\title{
Unsteady aerothermodynamics: Proposal for the establishment of a new branch within the discipline of aerothermodynamics
}

\author{
TONG BingGang \\ College of Physical Sciences, Graduate University of Chinese Academy of Sciences, Beijing 100080, China
}

Researchers from the College of Physical Sciences, GUCAS, have proposed to establish a new branch, unsteady aerothermodynamics, within the discipline of aerothermodynamics. The principal objectives of this new branch, to treat by theoretical means the study of physical phenomena relating to attached boundary layer flows, have been outlined in a preliminary investigation. A report based on a feasibility study has appeared in Vol. 54 No. 8 of Science China Physics, Mechanics \& Astronomy.

Aerothermodynamics, a cross-discipline based mainly on aerodynamics and thermodynamics, deals with hypersonic motion or high temperature gas flows and their interactions with objects, and in particular vehicles, travelling at hypersonic speeds. In itself, aerothermodynamics is of great importance in understanding aerodynamic and structural design of hypersonic vehicles, especially their thermal protection systems. However, traditionally in aerodynamics, according to simple oscillatory criterion characterized by the Strouhal number, engineers often ignore oscillatory flow mechanisms that give rise to the unsteadiness in vehicular aerothermodynamic environments. This stance has never been questioned and whether this criterion is appropriate within aerothermodynamics has never undergone critical assessment. The authors of this paper believe that this traditional thesis may not be strictly adequate and the application of aerothermodynamics to attached boundary layer flows should be studied from a more appropriate perspective that accounts for these unsteady flow mechanisms.

In this work, unsteady aerothermodynamics has been studied using simple attached boundary layer flow models and its principles explored in preliminary accounts using the governing equations for the flow. Analytic solutions to unsteady temperature fields and the unsteady wall heat fluxes were obtained for simple flow models and interaction characteristics between these unsteady temperature velocity fields were investigated exploiting these solutions. The authors have shown that unsteadiness in wall heat fluxes is dominated by two contributions: unsteady heat transfer originating from the temperature field and unsteady viscous dissipation originating from the velocity field. Subsequently, characteristics of the influence of unsteady velocity field on unsteady heat flux were discussed, and within the framework of these simplified models the main parameters describing these effects identified.

Furthermore, the authors derived similar criteria based on the compressible boundary layer equations that are valid within unsteady aerothermodynamics and discovered a new unsteadiness criterion for the temperature field. According to the dimensionless energy equation, the authors concluded that, rather than applying traditional methods developed for unsteady aerodynamics, the unsteadiness of flows in unsteady aerothermodynamics would be more comprehensively quantified by comparing the relative magnitudes of the unsteadiness criterion number newly encountered for the temperature field with coefficients of other terms in the dimensionless energy equation. To support their point of view, a numerical simulation of the unsteady boundary layer flow has been presented.

In this paper, the authors have proposed and initiated studies on phenomena falling within the new discipline of unsteady aerothermodynamics such as high speed flows. They also have suggested that further research should be instigated to better understand this special subject and its specific engineering challenges.

The authors all have backgrounds in aerothermodynamics developed within the research group lead by Professor TONG BingGang at GUCAS. This group focuses on fundamental theoretical studies on aerothermodynamic phenomena, including rarefied and real gas effects on hypersonic aerothermodynamics, physical criteria in computational aerothermodynamics, and unsteady aerothermodynamics.

Funding from the President Fund of GUCAS supported this research.

See the article: Chen H, Bao L, Tong B G. A theoretical study on the unsteady aerothermodynamics for attached flow models. Sci China Phys Mech Astron, 2010, 53: 1505-1514, doi.org/10.1007/s11433-010-4028-8 